\title{
Uterine Carcinosarcoma
}

National Cancer Institute

\section{Source}

National Cancer Institute. Uterine Carcinosarcoma. NCI Thesaurus. Code C42700.

A usually aggressive malignant neoplasm arising from the uterine corpus and less often the cervix. It is characterized by the presence of two components: a malignant epithelial component and a sarcomatous component. In the uterine corpus the epithelial component is usually glandular whereas in the cervix is usually non-glandular. Carcinosarcoma of the cervix, although it is aggressive, it may have a better prognosis compared to the uterine corpus carcinosarcoma. 